\title{
Proposta de um sistema de aprendizagem móvel com foco nas características e aplicações práticas da indústria 4.o
}

\author{
Eduardo dos Santos de Sá Carvalho¹, Nemésio Freitas Duarte Filho \\ edussc356@gmail.com,nemesio@ifsp.edu.br \\ ${ }^{1}$ Instituto Federal de Educação, Ciência e Tecnologia de São Paulo - IFSP, 14169263, Sertãozinho, Brasil
}

DOI: $10.17013 /$ risti.27.36-51

\begin{abstract}
Resumo: Sistemas de aprendizagem móvel surgem como uma importante alternativa de potencializar o ensino e a aprendizagem virtual. Neste contexto, a aprendizagem móvel vem sendo utilizada em diversas áreas do conhecimento, principalmente na área das Engenharias, flexibilizando e motivando o ensino e o treinamento por parte dos aprendizes e trabalhadores, sobretudo em relação as temáticas mais teóricas e abstratas. Deste modo, este trabalho tem o objetivo de desenvolver um sistema de aprendizagem móvel com foco nos conceitos e aplicações práticas da Industria 4.0. O sistema foi construído sob a forma web design responsivo, apresentando definições, terminologias, e um conteúdo educacional específico capaz de motivar e disseminar o conhecimento sobre o contexto da Indústria 4.0.
\end{abstract}

Palavras-chave: Industria 4.0; Aprendizagem Móvel; Sistema Educacional.

\section{Proposal for a mobile learning system focusing on the characteristics and applications practical of industry 4.0}

\begin{abstract}
Mobile learning systems emerge as an important alternative to enhance teaching and virtual learning. In this context, mobile learning has been used in several areas of knowledge, especially in the area of Engineering, making teaching and training more flexible and motivated by apprentices and workers, especially in relation to more theoretical and abstract themes. Therefore, this project aims to develop a mobile learning system focused on the concepts and guidelines of Industry 4.o. The system was constructed in the form of responsive web design, presenting definitions, terminologies, and a specific educational content capable of motivating and disseminating knowledge about the context of Industry 4.0.
\end{abstract}

Keywords: Industry 4.0; Mobile Learning; Educational System.

\section{Introdução}

Nos últimos anos, um novo contexto da indústria, denominada indústria 4.0 vem surgindo, revolucionando a forma de planejar e executar a manufatura industrial. 
A Indústria 4.0, integrada com aspectos tecnológicos, proporciona a execução de "Fábricas Inteligentes" com estruturas modulares, capazes de aumentar a produção e economizar custos.

De modo geral, a indústria 4.0 não é simplesmente um novo conceito, mas sim um conjunto de definições e tecnologias que em sinergia, podem proporcionar diversos benefícios na área industrial. Nesse contexto, por meio dos benefícios proporcionados pela aprendizagem móvel, já é possível observar e identificar iniciativas na literatura da utilização da aprendizagem móvel na área da Engenharia e Indústria (Bidin and Ziden, 2013; Ally, 2009; Liu, 2016), flexibilizando o ensino e o treinamento de aprendizes e trabalhadores. A aprendizagem móvel aplicada diretamente na indústria também proporciona a melhora na disseminação do conhecimento, em relação à novas tecnologias, conceitos, instruções, entre outros aspectos do dia a dia de um profissional da área.

Por ser um contexto novo, a indústria 4.0 possui carências em relação a informações, padronizações e conceitualizações. Algumas tecnologias ainda são abstratas em relação a sua aplicação prática, necessitando de um bom direcionamento e diretrizes de utilização. Um modelo educacional, desenvolvido sobre os preceitos da aprendizagem móvel pode possibilitar aos usuários contribuir, participar e acessar informações relevantes sobre esse novo contexto industrial através de dispositivos móveis, tais como celulares, i-pods, laptops, tv, entre outros, a qualquer momento e em qualquer lugar (Schepman et al. 2012).

Ressalta-se também, que além de modificações e adaptação nas indústrias, os profissionais (frente a esse novo contexto) também vão precisar se adaptar e atualizar (Gilchrist, 2016). Uma forma eficaz de se iniciar este processo é por meio da disseminação de informações/ conhecimento, entender e compreender (através de um conteúdo educacional lúdico) o que é a indústria 4.0, para que serve, e como poderá ser utilizada na prática.

Dentro desse contexto, este trabalho tem como objetivo desenvolver um sistema de aprendizagem móvel sobre as características e aplicações práticas da indústria 4.0, proporcionando uma melhora na disseminação do conhecimento desta área, ajudando alunos e profissionais. O sistema foi avaliado com a ajuda de especialistas (professores) e alunos, sendo identificados aspectos positivos e negativos (melhorias) em sua utilização prática.

O artigo encontra-se organizado como se segue. Na seção 2 é apresentado o referencial teórico, introduzindo conceitos sobre aprendizagem móvel e indústria 4.0. A seção 3 traz o planejamento e execução da revisão sistemática, forma que os autores utilizaram para sintetizar informações conceituais e exemplificações práticas sobre a indústria 4.0. A seção 4 apresenta a visão geral do sistema educacional, juntamente com as suas principais funcionalidades. A seção 5 mostra a validação do sistema. E por fim, na seção 6 temos a conclusão, juntamente com as perspectivas para trabalhos futuros.

\section{Referencial Teórico}

\subsection{Aprendizagem Móvel}

Nos últimos anos, temas relacionados ao ensino e aprendizagem têm sido discutidos e estudados pela comunidade científica. Os sistemas e ambientes de aprendizagem têm apresentando uma crescente importância tornando-se fundamental para o ensino e 
treinamento, sendo relevantes em ambientes acadêmicos, organizacionais e até mesmo dentro da área da indústria. Partindo deste raciocínio, muitas organizações e instituições estão cada vez mais utilizando sistemas de apoio ao ensino, proporcionando maior treinamento, disseminação do conhecimento e aperfeiçoamento de aprendizes, sejam eles pesquisadores, profissionais da área, ou interessados (Duarte Filho and Barbosa, 2015).

É possível verificar que o avanço tecnológico possibilitou uma nova realidade educacional. A integração entre as práticas educacionais juntamente com o avanço da tecnologia tem provocado uma mudança de comportamento nos aprendizes, professores e tutores em relação ao processo de ensino e aprendizagem (Svetlana, 2009). Um de seus efeitos é o aumento crescente da quantidade de informação disponível e acessível aos usuários.

Esses conceitos, juntamente com o advento e desenvolvimento da computação ubíqua, vêm proporcionando uma nova modalidade de ensino - a aprendizagem móvel ou mobile learning (m-learning) (Kinshuk, 2003; Nah, 2008; O’Malley, 2003; Wexler, 2008). A aprendizagem móvel caracteriza-se pela capacidade de proporcionar uma forte interação entre os aprendizes, professores e tutores, possibilitando aos mesmos contribuir, participar e acessar o ambiente de ensino por meio de dispositivos móveis (celulares, tablets, laptops, rádio, tv, entre outros).

Para proporcionar maior comodidade aos aprendizes e facilitar o acesso e utilização de materiais didáticos, a aprendizagem móvel utiliza-se das tecnologias de redes sem fio, Bluetooth, Wireless Application Protocol (WAP), General Packet Radio System (GPRS), Infra Red (IR) de recursos fornecidos pela telefonia celular, além das linguagens EXtensible Markup Language (XML) e Java, dos serviços de correio de voz e de mensagens curtas (SMS), da capacidade de transmissão de fotos, dos serviços de e-mail, mensagens multimídia (MMS) e vídeo sob demanda (Keegan, 2005).

Por se tratar de um paradigma novo e emergente, há diversas tentativas para se definir aprendizagem móvel. Koschembahr (2000) define aprendizagem móvel como qualquer tipo de ensino ou aprendizagem que ocorre quando o aluno não está em algum lugar fixo, ou quando o indivíduo se aproveita de oportunidades de aprendizagem disponibilizadas pelas tecnologias móveis, associando assim os conceitos tecnológicos e de mobilidade. Ozdamli and Cavus (2011) abordam aprendizagem móvel como uma atividade que permite aos indivíduos serem mais produtivos quando consomem, criam ou interagem com as informações, mediados por dispositivos digitais móveis e portáteis.

Este novo contexto de ensino além de facilitar a aprendizagem de seus usuários, pode também ser utilizado para apoiar o treinamento de trabalhadores no seu ambiente de trabalho. Assim os usuários trabalhadores podem passar mais tempo onde necessitam estar, e também dedicar o seu tempo à uma possível formação continuada (Koschembahr, 2000). Porém, por ser um modelo de aprendizagem emergente, os sistemas de aprendizagem móvel podem apresentar algumas limitações em sua utilização, como por exemplo: poder de processamento reduzido; tela de tamanho variado; energia limitada (dependente de baterias); comunicação com taxas de transmissão, geralmente, menores do que as das redes fixas; aspectos de usabilidade; entre outras (Xie, 2016).

Independentemente da definição adotada e eventuais limitações de uso, a utilização de sistemas de aprendizagem por meio de dispositivos móveis traz benefícios que vão além da acessibilidade, comodidade e comunicação (Schepman et al. 2012). Por exemplo, 
com dispositivos móveis os aprendizes podem utilizar aplicativos (processamento de texto, fotos), ambientes específicos de aprendizagem, acesso à Web, ferramentas de colaboração, redes sociais, adaptação ao contexto, feedback imediato, entre outros aspectos.

\subsection{Indústria 4.0}

O desenvolvimento, incorporação e aplicação de recentes inovações tecnológicas têm provocado mudanças sociais e econômicas. Estas mudanças, em acelerada expansão, alcançaram uma escala e escopo significativos, de modo que diversos estudos técnicos têm sugerido que estaríamos iniciando uma quarta revolução industrial, contexto este denominado "Indústria 4.0" (Gilchrist, 2016).

De acordo com Lee et al. (2015), a Indústria 4.0 é um conceito de indústria proposto recentemente e que engloba as principais inovações tecnológicas dos campos de automação, controle e tecnologia da informação, aplicadas aos processos de manufatura.

Entendendo a indústria 4.o como uma evolução dos sistemas produtivos industriais, podemos listar alguns benéficos previstos e já estudados e baseados no impacto nas organizações (Gilchrist, 2016; Lasi et al., 2014): (1) Redução de Custos; (2) Economia de Energia; (3) Aumento da Segurança; (4) Conservação Ambiental; (5) Redução de Erros; (6) Fim do Desperdício; (7) Transparência nos Negócios; (8) Aumento da Qualidade de Vida; (9) Personalização e Escala sem Precedentes, entre outros.

Quando dizemos que a internet está na indústria, no meio produtivo, devemos pensar num ambiente onde todos os equipamentos e máquinas estão conectadas em redes e disponibilizando informações de forma única, esse conceito é chamado de Internet das Coisas (IoT - Internet of Things) (Lee et al., 2015).

A partir de Sistemas Cyber-Físicos, Internet das Coisas e Internet dos Serviços, os processos de produção tendem a se tornar cada vez mais eficientes, autônomos e customizáveis. Isso significa um novo período no contexto das grandes revoluções industriais (Lee et al., 2015). Com as fábricas inteligentes, diversas mudanças ocorrerão na forma em que os produtos serão manufaturados, causando impactos em diversos setores do mercado.

A tecnologia base responsável por este conceito é o IoT - Internet of Things (Internet das Coisas) e o M2M - Machine to Machine (Máquina para Máquina). A Internet das Coisas, como comentado anteriormente é a conexão lógica de todos os dispositivos e meios relacionados ao ambiente produtivo em questão, os sensores, transmissores, computadores, células de produção, sistema de planejamento produtivo, diretrizes estratégicas da indústria, informações de governo, clima, fornecedores, tudo sendo gravado e analisado em um banco de dados (Lee et al., 2015).

Existem seis princípios (Brettel and Rosenberg, 2014) para o desenvolvimento e implantação da indústria 4.o, que definem os sistemas de produção inteligentes que tendem a surgir nos próximos anos. São eles:

- Capacidade de operação em tempo real: consiste na aquisição e tratamento de dados de forma praticamente instantânea, permitindo a tomada de decisões em tempo real. 
- Virtualização: simulações já são utilizadas atualmente, assim como sistemas supervisórios. No entanto, a indústria 4.0 propõe a existência de uma cópia virtual das fabricas inteligentes. Permitindo a rastreabilidade e monitoramento remoto de todos os processos por meio dos inúmeros sensores espalhados ao longo da planta.

- Descentralização: a tomada de decisões poderá ser feita pelo sistema cyberfísico de acordo com as necessidades da produção em tempo real. Além disso, as máquinas não apenas receberão comandos, mas poderão fornecer informações sobre seu ciclo de trabalho. Logo, os módulos da fábrica inteligente trabalharão de forma descentralizada a fim de aprimorar os processos de produção.

- Orientação a serviços: utilização de arquiteturas de software orientadas a serviços aliado ao conceito de Internet of Services.

- Modularidade: produção de acordo com a demanda, acoplamento e desacoplamento de módulos na produção. O que oferece flexibilidade para alterar as tarefas das máquinas facilmente.

\section{Revisão Sistemática}

Revisão Sistemática é uma metodologia utilizada para realizar revisões bibliográficas de forma organizada, possibilitando avaliar e interpretar todos os trabalhos relevantes disponíveis sobre um determinado assunto. As Revisões Sistemáticas têm o objetivo de apresentar uma avaliação justa de um tema de pesquisa, proporcionando maior fundamentação teórica e confiabilidade científica (Kitchenham, 2004).

A revisão sistemática, neste trabalho, foi utilizada para selecionar os principais conceitos sobre indústria 4.0, juntamente com artigos que analisassem aplicações práticas e características da indústria 4.o. Todas as informações coletadas foram sintetizadas em um sistema de aprendizagem móvel baseada nesse contexto.

Levando em consideração o contexto de execução deste trabalho, na etapa de planejamento foi estabelecido o protocolo da revisão, deste modo, foram propostas e levantadas questões relacionadas à pesquisa, sendo feita a seleção das fontes de informações e, por fim, a seleção dos estudos. A Tabela 1 resume os objetivos principais e secundários da pesquisa.

\section{Objetivos Principais}

Identificar os principais estudos de casos e aplicações práticas no contexto da indústria 4.0.

\section{Objetivos Secundários}

Verificar as principais dificuldades e limitações das as indústrias com relação à implementação das novas tecnologias na indústria 4.0.
Identificar as principais tecnologias, padrões e conceitos da indústria 4.0.
Analisar onde e quando vem sendo utilizadas as novas ideias da indústria 4.0.

Tabela 1 - Objetivos Principais e Secundários

As questões para a pesquisa foram levantadas da seguinte forma (Tabela 2). 


\section{Questões}

Questão 1: Existem estudos de casos e aplicações práticas no contexto da indústria?

Questão 2: Quais as principais tecnologias, conceitos e padrões da indústria 4.0 utilizados em um contexto real?

Questão 3: Quais são os principais requisitos para a implementação da indústria 4.o no meio?

Questão 4: Quais os principais aspectos temporais e de localidade que estão sendo utilizados na aplicação prática da indústria 4.0 ?

Tabela 2 - Questões da Pesquisa

Depois de estabelecido as questões de pesquisa, foram definidas as estratégias de busca. Para isso, foram definidos critérios para a seleção das fontes de busca, palavraschaves e strings de busca. Alguns critérios foram definidos em relação às fontes de busca, proporcionando a coleta de informações relevantes ao tema da pesquisa. Fontes de busca foram escolhidas levando em consideração o impacto e a abrangência na área da "Indústria" e "Engenharias". A partir dos critérios analisados, foram escolhidas como fontes de pesquisa: IEEE Xplore, ACM Digital Library, Springer Link, Science Direct (Scirus), Scopus, ISI Web Knowledge (Web of Science), Google Scholar, EI Compendex.

Critérios de inclusão e exclusão também foram definidos para esta pesquisa. A Tabela 3 sintetiza os critérios estabelecidos.

\section{Critérios de Inclusão (CI)}

Estudos primários que mostram aplicações práticas e análises de estudos de casos no contexto da indústria 4.0

\section{Critérios de Exclusão (CE)}

Estudos primários que evidenciam aplicações da indústria 4.0 sem embasamento prático ou real
Estudos primários que destacam características e conceitos utilizados em um contexto real da indústria 4.0

Estudos primários em que a indústria 4.0 seja aplicada no meio de produção
Estudos primários que estejam em idiomas diferentes do inglês e português, dificultando a coleta e a identificação de informações

Estudos primários que não estejam disponíveis para download na base de dados, pois para uma análise eficiente todo o documento deve ser lido

Tabela 3 - Critérios de Inclusão e Exclusão

Na etapa seguinte, o processo de construção da string de busca exige atenção, já que sua definição está ligada diretamente aos objetivos, escopo e resultados da pesquisa. Antes da definição da string de busca, testes foram realizados nas próprias bases de dados, utilizando alguns sinônimos definidos nas palavras-chaves, verificando a forma e a quantidade de resultados obtidos. Tendo como base os testes realizados, a string genérica utilizada nas fontes de pesquisa foi definida da seguinte forma:

$$
\begin{gathered}
\text { ("Industry 4.O” OR "Smart Industry" OR "Smart Manufacturing” OR "Fourth } \\
\text { Industrial Revolution") AND ("Case study" OR "Practical Application" OR } \\
\text { "Real Applications") }
\end{gathered}
$$


Tendo como base a string genérica foram construídas strings específicas para cada base de dados. As buscas foram realizadas a partir das palavras-chaves, sendo inseridas no título e no abstract dos trabalhos em relação às bases de dados definidas anteriormente.

A partir das buscas realizadas, 213 documentos foram retornados. Após a análise, foram selecionados 129 trabalhos, os quais estavam integralmente disponíveis para leitura (Tabela 4).

\begin{tabular}{lll}
\hline Base de Dados & Resultado Total & Resultado pós Análise \\
\hline ACM Digital Library & 3 & 2 \\
\hline EI Compendex & 25 & 1 \\
\hline Google Scholar & 66 & 66 \\
\hline IEEE Xplore & 34 & 34 \\
\hline ISI web knowledge (Web of Science) & 0 & o \\
\hline Science Direct (Scirus) & 14 & 8 \\
\hline Scopus & 36 & 0 \\
\hline Springer Link & 35 & 18 \\
\hline Resultado Final & 213 & 129 \\
\hline
\end{tabular}

Tabela 4 - Resultado Parcial da Revisão Sistemática

Após a leitura dos resumos dos 129 estudos primários e a aplicação dos critérios de inclusão e exclusão, o número de trabalhos foi reduzido para 20. A relação das bases de dados e artigos selecionados é sintetizada na Tabela 5 .

Os 20 estudos foram lidos e analisados em conformidade com os objetivos e as questões definidas previamente. Para cada trabalho analisado os autores identificaram características importantes para uma aplicação educacional com foco na indústria 4.o, sintetizando principalmente elementos relacionados as características, definições e exemplificações praticas da indústria.

\section{Sistema de Aprendizagem Móvel com foco na Indústria 4.o}

Após a síntese e análise de toda a informação sobre a indústria 4.o, foi feito o desenvolvimento da parte prática, ou seja, partindo para o desenvolvimento de um sistema computacional. O desenvolvimento foi realizado utilizando tecnologias web, proporcionando maior portabilidade e adequações aos dispositivos móveis. Sendo identificados os principais requisitos funcionais e não funcionais do sistema educacional.

O conteúdo educacional (sendo incorporado ao sistema computacional) foi desenvolvido com as informações e análises das pesquisas realizadas anteriormente, ela foi organizada de forma simples e lúdica, utilizando uma ordem crescente de informações (desde aspectos introdutórios, até as conclusões sobre temáticas da indústria 4.0). Para melhorar o entendimento, de forma didática, foram utilizados diversos recursos multimídia: 


\begin{tabular}{|c|c|c|}
\hline ID & Título do Trabalho & Fonte \\
\hline 1 & A Modular Web Framework for Socio-CPS-Based Condition Monitoring & IEEE \\
\hline 2 & $\begin{array}{l}\text { Attribute identification and predictive customisation using fuzzy clustering and } \\
\text { genetic search for Industry } 4.0 \text { environments }\end{array}$ & Google Scholar \\
\hline 3 & $\begin{array}{l}\text { Big data oriented macro-quality index based on customer satisfaction index } \\
\text { and PLS-SEM for manufacturing industry }\end{array}$ & IEEE \\
\hline 4 & $\begin{array}{l}\text { Big-data-driven anomaly detection in industry -4.0- An approach and a case } \\
\text { study }\end{array}$ & IEEE \\
\hline 5 & Brushless DC motors as parts of Smart Factories & IEEE \\
\hline 6 & $\begin{array}{l}\text { Collaborative maintenance in flow-line manufacturing environments An } \\
\text { Industry } 4.0 \text { approach }\end{array}$ & Science Direct \\
\hline 7 & Cyber-Integrated Big Data Analytics Agent for Industry 4.o Applications & Google Scholar \\
\hline 8 & Cyber-Physical System for maintenance in industry 4.0 & Google Scholar \\
\hline 9 & $\begin{array}{l}\text { Cyber-physical Systems Architecture for Self-Aware Machines in Industry } 4.0 \\
\text { Environment }\end{array}$ & Science Direct \\
\hline 10 & Design Principles for Industrie 4.o Scenarios & IEEE \\
\hline 11 & Disruption-free software updates in automation systems & IEEE \\
\hline 12 & $\begin{array}{l}\text { Identifying Smart Design Attributes for Industry 4.o Customization Using a } \\
\text { Clustering Genetic Algorithm }\end{array}$ & Google Scholar \\
\hline 13 & Importance_of_Production_Environments_When_Applyin & Google Scholar \\
\hline 14 & Improving maintenance processes with distributed monitoring systems & IEEE \\
\hline 15 & Industrial Maintenance with Augmented Reality Two Case Studies & $\begin{array}{l}\text { ACM Digital } \\
\text { Library }\end{array}$ \\
\hline 16 & Industry 4.0 Applications - Case Study of Fortune Electric & Google Scholar \\
\hline 17 & $\begin{array}{l}\text { Integration of agent technology into manufacturing enterprise A review and } \\
\text { platform for industry } 4.0\end{array}$ & IEEE \\
\hline 18 & Production related IT solutions in the operation of factories & IEEE \\
\hline 19 & $\begin{array}{l}\text { Self-organizing tool for smart design with predictive customer needs and wants } \\
\text { to realize Industry } 4.0\end{array}$ & IEEE \\
\hline 20 & $\begin{array}{l}\text { Visualization of RFID-enabled shopfloor logistics Big Data in Cloud } \\
\text { Manufacturing }\end{array}$ & Springer Link \\
\hline
\end{tabular}

Tabela 5 - Resultado Final da Revisão Sistemática

imagens, vídeos, gifs e outros elementos. Também foram inseridos exercícios/testes para uma melhor fixação do conhecimento.

O sistema foi desenvolvido para atingir diferentes tipos de públicos, como estudantes interessados no assunto, professores da área de engenharia, trabalhadores do setor industrial, ou até mesmo para pessoas leigas que estão indo em busca do conhecimento e se interessam pelo tema abordado. 


\subsection{Processo de Desenvolvimento}

O processo de desenvolvimento do sistema educacional foi definido nas seguintes etapas.

1. Concepção e Definição do Escopo: a definição da problemática surgiu a partir das necessidades e carências encontradas na comunidade e no meio científico, e também por se tratar de um tema recente. Muitos embasamentos teóricos da indústria 4.0 não são demonstrados na prática, se tornando um tema mais teórico e abstrato, dificultando o seu entendimento por parte dos alunos.

2. Estudo dos Materiais: para uma melhor coleta e direcionamento dos materiais foi conduzida uma revisão sistemática, possibilitando a coleta de diferentes artigos relevantes e confiáveis, dentro do tema desejado. Os artigos finais (após aplicação dos critérios de inclusão e exclusão) foram lidos por completo, sintetizando informações relevantes ao contexto educacional.

3. Identificação e estudo de tecnologias para o desenvolvimento de aplicações móveis: após a síntese e análise de toda a informação sobre a indústria 4.0 e aprendizagem móvel, foi feito o desenvolvimento da parte prática. $\mathrm{O}$ sistema foi desenvolvido utilizando a ferramenta Phonegap ${ }^{1}$, possibilitando a criação de um sistema híbrido e ao mesmo tempo portável e responsivo em diferentes plataformas mobile. A linguagem de programação utilizada foi o HTML $5^{2}$, e consequentemente outras bases de programação web foram utilizadas: o $\mathrm{CSS}_{3}^{3}$ e o JavaScript ${ }^{4}$. Com o HTML 5 foi feita toda a parte de estruturação e nomenclatura do programa, já o CSS 3 ajudou na definição do estilo do sistema, e por fim, o JavaScript foi utilizado para reproduzir pequenas animações e eventos em relação a parte do cliente da aplicação.

4. Identificação e seleção dos tópicos a serem explorados: os tópicos educacionais do sistema foram elaborados da seguinte maneira: (1) início, (2) introdução, (3) pilares da indústria 4.o, (4) exemplos práticos, (5) exercícios, e (6) bibliografias. Essa ordem foi definida para proporcionar maior flexibilidade e dinamismo aos alunos. Ressalta-se também que dentro dessa estrutura também foram inseridos subitens para melhor dividir e segmentar as informações educacionais no sistema.

5. Avaliação: para demonstrar a aplicabilidade prática do sistema educacional, foram realizados testes e atividades com professores e alunos, possibilitando a identificação de limitações e possíveis melhorias no sistema.

\subsection{Principais Características e Funcionalidades}

O sistema foi implementado procurando incorporar interações de usabilidade (Figura 1), visando atingir o maior número de usuários e também uma maior facilidade de acesso a eles, sendo elas:

- Web Responsivo(Figura 1.a): pode-se dizer que uma das principais características desta aplicação prática é a responsividade, pois a aplicação consegue se

\footnotetext{
${ }^{1}$ https://phonegap.com/

${ }^{2}$ https://www.w3schools.com/html/

3 https://www.w3schools.com/css/

${ }^{4}$ http://tableless.github.io/iniciantes/manual/js/
} 
adequar a diversos dispositivos móveis, ou tamanhos de telas diferentes, sem descaracterização ou perdas de informações;

- Informações Complementares (Figura 1.b): para que não haja uma sobrecarga cognitiva frente aos usuários do sistema em relação a leitura em um dispositivo móvel, o sistema educacional utiliza recursos para "ocultar informações complementares", por meio de botões, onde o aprendiz pode acessar quando quiser e quando achar necessário as informações adicionais do texto.

- Recurso Multimídia (Figura 1.c): para efeitos lúdicos e de exemplificações, foram utilizados vários recursos multimídia, como por exemplo: imagens, vídeos, gifs, e também links externos;

- Segmentação de Temas (Figura 1.d): Os temas da aplicação foram segmentados, tentando contemplar um histórico sobre o contexto da indústria 4.0, levando em consideração as seguintes temáticas: início, introdução, pilares da indústria 4.o, exemplos práticos, exercícios e bibliografias. Para cada tópico, foram inseridos subitens/divisões que possibilita o leitor a continuar a sua leitura em momentos distintos. Para isso, foi criada uma "paginação"

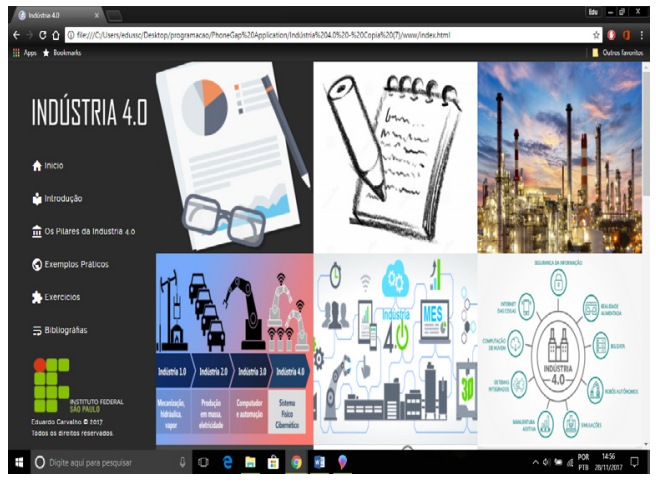

(a) Web Responsivo

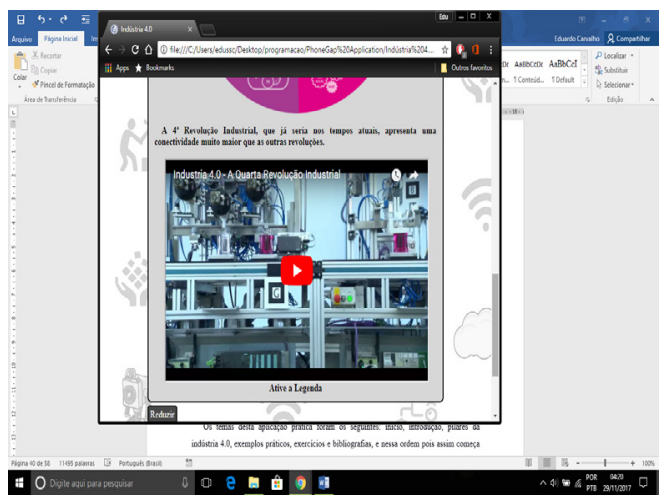

(c) Recurso Multimídia

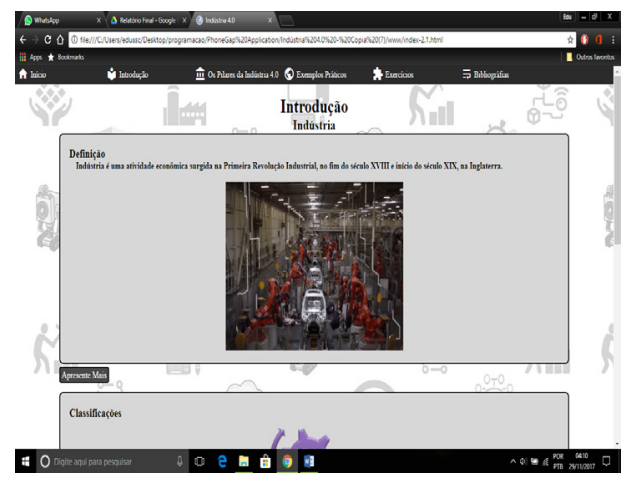

(b) Informações Complementares

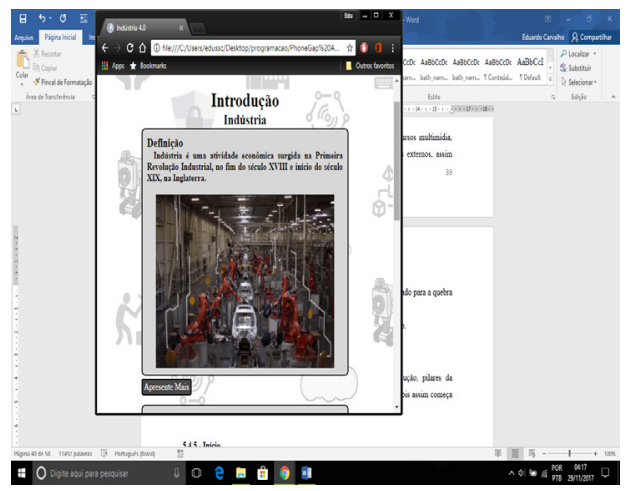

(d) Segmentação de Temas

Figura 1 - Aspectos de Usabilidade do Sistema 
segmentada, possibilitando o leitor avançar e retroceder quando ele quiser em relação a um determinado tópico do sistema educacional.

Como ressaltado anteriormente, as temáticas do conteúdo do sistema foram divididas em seções menores para um melhor entendimento, tendo os seguintes objetivos e descrições (Figura 2):

- Início (Figura 2.a): o início, ou a capa, possui basicamente dois menus de acesso ao usuário, um mais simples, apenas com os principais tópicos do sistema, e um

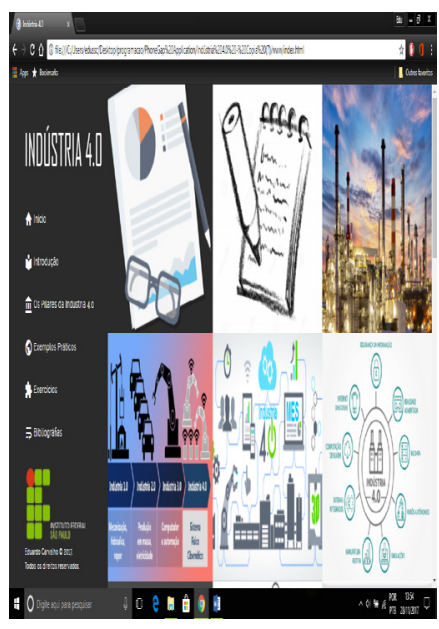

(a) Inicio

\section{MENU lä}

\section{Exemplos Práticos}

Temas

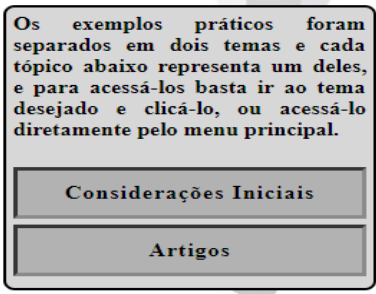

Autor: Eduardo Carvalho e 2017. Todos os direitos reservados. direitos rese

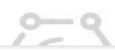

(d) Exemplos Práticos

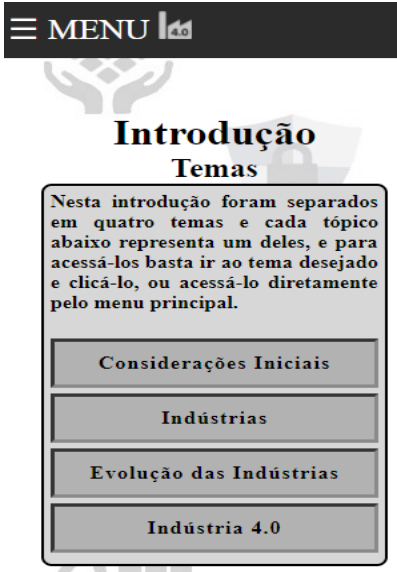

Autor: Eduardo Carvalho $\odot$ 2017. Todos os

(b) Introdução

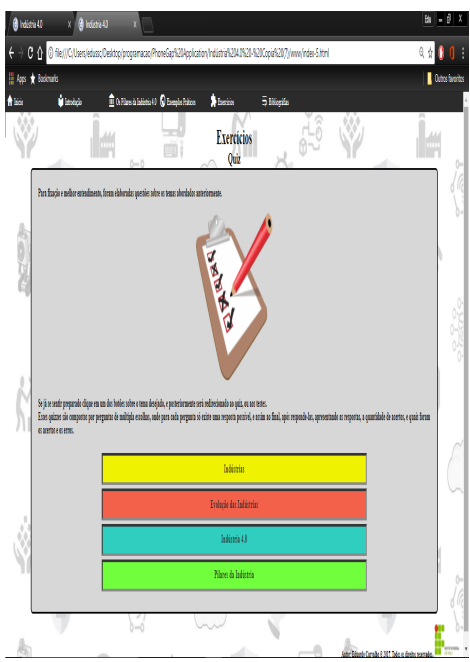

(e) Exercícios

\section{MENU lato}

\section{Os Pilares da Indústria}

\section{0}

\section{Temas}

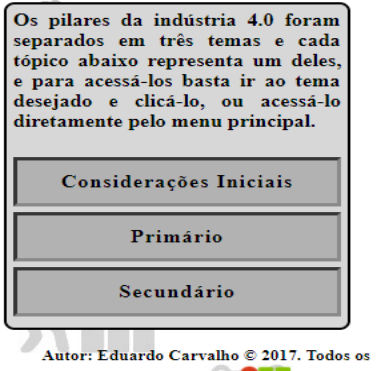

ofits

(c) Pilares da Indústria 4.0

\section{MENU lad}

\section{Bibliográfias}

Hans Fleischmann, Johannes Kohl,
Jörg Franke (2016), A Modular
Web Framework for Socio-CPS-
Based Condition Monitoring.
Alfredo Alan Flores Saldivar, Cindy
Goh, Yun Li, Hongnian Yu, Yi
Chen (15/12/2016), Attribute
identification and predictive
customisation using fuzzy
clustering and genetic search for
Industry 4.0 environments.
Tao Li, Yihai He, Chunling Zhu
(2016), Big data oriented macro-
quality index based on customer
satisfaction index and PLS-SEM for
manufacturing industry.
Ljiljana Stojanovic Fraunhofer,
Marko Dinic, Nenad Stojanovic,
Aleksandar Stojadinovic (2016),
Dindatndvivnn Annmalv nntnotinn

(f) Bibliografias

Figura 2 - Segmentação do conteúdo educacional 
menu mais complexo com imagens (clicáveis), descrição da versão do sistema e uma sintetização de todos os temas apresentados na aplicação educacional;

- Introdução (Figura 2.b): a introdução é composta por subtemas: indústrias (o que é), evolução das indústrias (histórico e exemplos) e indústria 4.0 (apresentação de novos conceitos). Para cada tema foi realizado uma breve apresentação e análise, apresentando conceitos/definições, classificações, comparações e outros aspectos;

- Pilares da Indústria 4.o (Figura 2.c): os pilares da indústria 4.0 foram separados em dois grandes grupos, facilitando a análise e o entendimento por parte dos alunos. Nestes grupos apresentamos elementos relacionados diretamente com aspectos tecnológicos, big data, internet das coisas, conectividade, adaptação ao contexto, entre outros fatores aplicados na indústria 4.0;

- Exemplos Práticos (Figura 2.d): esta seção apresenta exemplificações práticas da indústria 4.0, no mundo atual, juntamente com características e curiosidades. Todas as exemplificações foram tiradas dos artigos analisados, tendo como objetivo mostrar ao leitor as inúmeras possibilidades de aplicações da indústria 4.0 na atualidade.

- Exercícios (Figura 2.e): como forma de fixação do conhecimento adquirido, foram desenvolvidos exercícios na modalidade de questões fechadas. Os exercícios foram divididos em quatro grupos, com várias questões de múltiplas escolhas. Cada pergunta possui apenas uma resposta correta, e ao efetuar a respostas, o sistema emite um feedback instantâneo, mostrando a resposta correta e uma breve explicação em relação ao erro ou acerto da resposta.

- Bibliografia (Figura 2.f): por fim, foi elaborada uma seção onde foi inserida toda a bibliografia utilizada, permitindo que o leitor, sendo ele aluno ou professor, possa se aprofundar na contextualização da indústria 4.0.

\section{Validação}

No contexto da validação do sistema educacional, foram utilizados alunos dos cursos "Tecnológico em Automação Industrial", "Engenharia Elétrica" e "Engenharia Mecânica"; e professores especialistas na área de Engenharia. A participação e feedback dos participantes em uma avaliação prática do sistema é importante ao trabalho, pois proporciona um conjunto de críticas (positivas e negativas), garantindo melhorias e ajustes tanto em um nível técnico quanto educacional e pedagógico.

Para a realização desta validação, um grupo de 68 alunos receberam atividades a serem realizadas no sistema de aprendizagem móvel com foco no contexto da Industria 4.0. Todas as atividades realizadas com os alunos foram instruídas e orientadas pelos autores do trabalho. Os alunos realizaram as seguintes atividades:

1. Baixar, instalar e acessar o sistema educacional: nesta atividade foi verificado a facilidade de instalação e acesso do sistema em diferentes dispositivos móveis. Os alunos tiveram à sua disposição diferentes dispositivos (cedidos pela faculdade), podendo também acessar a aplicação com o seu próprio aparelho móvel. Nenhum treinamento prévio foi realizado aos alunos (pois o objetivo foi verificar a facilidade de início em seu acesso e utilização). O que foi definido, 
foi apenas o local do repositório do sistema (link), e algumas informações sobre o objetivo do sistema e do experimento.

2. Iniciar e finalizar um módulo do sistema: o sistema possui vários módulos educacionais, segmentando informações introdutórias e avançadas sobre a Industria 4.o. Nesta atividade, os alunos foram instruídos para escolherem um dos módulos e realizar a sua execução do início ao fim, passando por todos os slides e recurso educacionais (imagens, vídeos, infográficos, entre outros).

3. Execução do simulado relacionado ao módulo estudado: o objetivo desta atividade foi verificar a fixação do conhecimento proporcionado pelo sistema educacional. Para isso, os alunos realizaram uma bateria de questões referentes ao módulo que foi executado/estudado na etapa anterior (ao final de cada módulo, o sistema disponibiliza uma bateria de questões na forma de simulado, referente ao conteúdo estudado, dando um feedback em relação as respostas certas e erradas). Nesta atividade, além do critério de fixação do conhecimento, outros critérios também foram avaliados, sendo eles: feedback das questões, organização, estrutura, pontuações, entre outros aspectos educacionais.

4. Aplicação de um questionário: ao final da execução das atividades, os alunos responderam a um questionário online sobre diversos critérios do sistema educacional. O questionário teve como objetivo coletar informações sobre a percepção de uso do sistema por partes dos alunos, podendo os mesmos incorporar aspectos positivos e negativos da aplicação. As questões em sua maioria, possuíam opções de respostas em escala: Muito satisfatório, Satisfatório, Regular, e Insatisfatório.

O questionário online foi aplicado ao término da realização das atividades, sendo composto por critérios (Acesso, Módulo Educacional, Simulado e Informações Complementares). Para cada critério foram incorporadas questões de múltipla escolha para facilitar o seu preenchimento. A Tabela 6 apresenta os resultados obtidos juntamente com o número de itens e critérios de avaliação.

Além disso, ressalta-se que as informações complementares se referem a questões adicionais com campos editáveis (perguntas abertas), que permitiram aos participantes inferir pontos positivos/negativos. Nas Tabelas 6 e 7, a pontuação das informações complementares é apresentada como NA (Não se aplica).

\begin{tabular}{lllll}
\hline Critério & $\begin{array}{l}\text { Muito } \\
\text { Satisfatório }\end{array}$ & Satisfatório & Regular & Insatisfatório \\
\hline Acesso do Sistema (10 itens) & $69 \%$ & $\mathbf{2 5 \%}$ & $\mathbf{4 , 5} \%$ & $\mathbf{1 , 5} \%$ \\
\hline Módulo Educacional (9 itens) & $76,5 \%$ & $19 \%$ & $\mathbf{1 , 5} \%$ & $3 \%$ \\
\hline Simulado de Avaliação (9 itens) & $67,5 \%$ & $26,5 \%$ & $3 \%$ & $3 \%$ \\
\hline $\begin{array}{l}\text { Informações Complementares (3 } \\
\text { itens) }\end{array}$ & $\mathrm{NA}$ & $\mathrm{NA}$ & $\mathrm{NA}$ & $\mathrm{NA}$ \\
\hline
\end{tabular}

Tabela 6 - Resultado do questionário com alunos.

De modo complementar, ao longo da validação do sistema, os autores verificaram também a necessidade de sintetizar a visão crítica do professor da área, sobre a utilização 
do sistema educacional, principalmente em níveis pedagógicos e educacionais. O procedimento aplicado aos professores foi semelhante ao aplicado aos alunos, porém, com atividades e questões diferenciadas.

Ao final, foi realizado o preenchimento de um formulário online. Este procedimento foi realizado com a participação de 6 docentes. Destaca-se que os professores participantes são da área de Engenharia, possuindo um conhecimento prévio sobre o contexto da Industria 4.0. A Tabela 7 sintetiza os resultados obtidos pela avaliação dos docentes.

\begin{tabular}{lllll}
\hline Critério & $\begin{array}{l}\text { Muito } \\
\text { satisfatório }\end{array}$ & Satisfatório & Regular & Insatisfatório \\
\hline Estrutura e Organização (7 itens) & $67 \%$ & $16,5 \%$ & $16,5 \%$ & $0 \%$ \\
\hline $\begin{array}{l}\text { Informação Educacional } \\
\text { (12 itens) }\end{array}$ & $50 \%$ & $33,5 \%$ & $16,5 \%$ & $0 \%$ \\
\hline $\begin{array}{l}\text { Informações Complementares } \\
\text { (4 itens) }\end{array}$ & $\mathrm{NA}$ & $\mathrm{NA}$ & $\mathrm{NA}$ & $\mathrm{NA}$ \\
\hline
\end{tabular}

Tabela 7 - Resultado do questionário com docentes da área.

De modo geral, é possível concluir que o sistema educacional móvel com foco no contexto da indústria 4.0 apresenta uma boa aceitação tanto por parte dos alunos quanto professores, possuindo níveis considerados aceitáveis frente a critérios de acesso, utilização via dispositivos móveis, conteúdo, feedback de informações, elementos didáticos/pedagógicos, gerenciamento, entre outros aspectos.

Após a análise dos dados, principalmente das questões abertas "Informações Complementares”, foi possível observar que tanto alunos quanto professores tiveram pontos semelhantes e convergentes em relação a aspectos de melhoria do sistema, sendo eles: (1) ausência de um tutorial auto explicativo (com vídeos e animações), que pudesse ajudar leigos na utilização inicial do sistema educacional; (2) ausência de múltiplos idiomas. A primeira versão do sistema apresenta apenas o idioma português; (3) ausência de aspetos ligados a acessibilidade, principalmente relacionados com pessoas com necessidades especiais (visuais, auditivas, entre outras); e (4) falta de funcionalidade para que o professor possa inserir novas informações. Todas as críticas/melhorias estão sendo analisadas e algumas já estão em desenvolvimento.

\section{Conclusões}

No contexto da indústria 4.0, verifica-se uma carência e necessidade de aplicações simples e flexíveis, que proporcionem uma aprendizagem adequada por meio de dispositivos móveis e que, ao mesmo tempo, ajude no entendimento e ensino dos conceitos e aplicabilidade prática da indústria 4.0.

Devido a esta carência, a pesquisa teve como objetivo a proposta e o desenvolvimento de um sistema de aprendizagem móvel, objetivando promover uma formação continuada aos aprendizes e demais interessados que buscam conhecimentos sobre o tema da indústria 4.o, tendo um cenário lúdico, didático e com foco principalmente nos conceitos e características da indústria 4.o. 
O sistema educacional é direcionado para usuários em sua maioria graduandos (jovens entre 18 e 30 anos, principalmente dos cursos de engenharias e afins), sendo também útil para profissionais da área da indústria e pesquisadores. Todos estes podem utilizar o sistema para consultar informações e atualizar seus conceitos sobre temas específicos à indústria 4.0

O sistema foi desenvolvido sendo capaz de sintetizar diversas informações sobre o conceito de indústria 4.o, proporcionando um repositório educacional para o tema proposto. Possui uma interface amigável com diversas ferramentas direcionadas à aprendizagem, possibilitando um conteúdo didático adequado. Ao longo do desenvolvimento foram consideradas características de usabilidade a serem incluídas no sistema, visando maior flexibilidade, motivação, adaptação e percepção por parte dos aprendizes.

Ao longo da avaliação do sistema, através da aplicação de um questionário com especialistas e alunos da área, foram observados pontos positivos/negativos em relação a utilização em um contexto real. Os pontos limitantes identificados ao longo das avaliações foram: (1) ausência de tutoriais/manuais de uso; (2) falta de aspectos de acessibilidade; e (3) ausência de funcionalidade de gerenciamento do professor, possibilitando o mesmo fazer inserções de novos conteúdos.

Como trabalhos futuros, pretende-se modificar e evoluir o sistema de aprendizagem móvel, garantindo maior possibilidade de colaboração e contribuição entre os aprendizes. Para isso, um fórum/blog já vem sendo desenvolvido, incorporando dentro do sistema uma maior colaboração entre os seus usuários. Em segundo plano, será realizado a adição de novos conteúdos, novidades e atualizações sobre a indústria 4.0, permitindo que o sistema evolua, vendo que a temática abordada é atual e emergente.

\section{Referências}

Ally, M. (2009). Mobile learning: transforming the delivery of education and training. Athabasca: Athabasca university press.

Bidin, S., \& Ziden, A. A. (2013). Adoption and application of mobile learning in the education industry. Procedia-social and behavioral sciences, 90, 720-729.

Brettel, M., Friederichsen, N., Keller, M., \& Rosenberg, M. (2014). How virtualization, decentralization and network building change the manufacturing landscape: an industry 4.0 perspective. International journal of mechanical, industrial science and engineering, 8(1), 37-44.

Duarte Filho, N. F., \& Barbosa, E. F. (2015). A Contribution to the Establishment of Reference Architectures for Mobile Learning Environments. IEEE-RITA, 10, 234-241.

Gilchrist, A. (2016). Introducing industry 4.0. in Industry 4.0 (pp. 195-215). New York: apress.

Keegan, D. (2005). The incorporation of mobile learning into mainstream education and training. In: Proccedings of mlearning 2005-4th world conference on m-learning, Cape Town, 2005. 
Kinshuk, T., Suhonen, J., Sutinen, E., \& Goh, T. (2003). Mobile technologies in support of distance learning. Asian journal of distance education, 1(1), 60-68.

Koschembahr, C. (2005). Mobile learning: the next evolution. Chief learning officer, february.

Lasi, H., Fettke, P., Kemper, H. G., Feld, T., \& Hoffmann, M. (2014). Industry 4.o. Business \& information systems engineering, 6(4), 239.

Lee, J., Bagheri, B., \& Kao, H. A. (2015). A cyber-physical systems architecture for industry 4.o-based manufacturing systems. Manufacturing letters, 3, 18-23.

Liu, X. (2016). The application of multi-modal food industry teaching mode to mobile learning under cloud environment. Advance journal offood science and technology, 10(1), 77-80.

Ozdamli, F., \& Cavus, N. (2011). Basic elements and characteristics of mobile learning. World conference on educational technology researches, 28, 937-94.

Schepman, A., Rodway, P., Beattie, C., \& Lambert, J. (2012). An observational study of undergraduate students adoption of (mobile) note-taking software. Computers in human behavior, 28(2), 308-317.

Shi, R. (2016). Research on Effectiveness of Mobile English Teaching Software to Listening Learning of College Students. RISTI - Revista Ibérica de Sistemas e Tecnologias de Informação, (E13), 184.

Xie, Y. (2016). On teaching reform of college English based on mobile learning. RISTI Revista Ibérica de Sistemas e Tecnologias de Informação, (E9), 214. 